\title{
Poynting Vectors in Geomagnetic and Geoelectric Variations
}

\author{
Izumi YOKOYAMA* \\ Department of Geophysics, Faculty of Science, Hokkaido University, Sapporo 060, Japan
}

(Received August 25, 1987; Revised October 27, 1987)

\section{Introduction}

Poynting vectors defining the energy flows in electromagnetic fields are introduced into the studies of geomagnetic and geoelectric variations associated with polar substorms. In this paper the Poynting vectors are defined particularly by horizontal components of both the fields because the energy flow is estimated vertically to the earth surface, and the geomagnetic vertical components are compared with the geomagnetic horizontal components. Characteristics of the vectors are exemplified by the observations at Kakioka and Memanbetsu Geomagnetic Observatories, both belonging to the Japan Meteorological Agency, which show different manners in geomagnetic and geoelectric variations. Generally the Poynting vectors attain the maximum when the magnetic and electric fields are perpendicular to each other. As an observational result, the vertical component of a geomagnetic variation takes the maximum whether it is positive or negative when the Poynting vector of the variation takes the maximum. Considering that geoelectric variations have a preferred horizontal direction characteristic of each station, one may say that anomalous behaviors of the vertical component of geomagnetic variations which undergo mainly "conductivity anomaly", bear a relation with anisotropy of geoelectric variations and further with that of earth conductivity.

2. Examples of Geomagnetic and Geoelectric Variations Associated with Polar Substorms

In this paper, magnetograms and geoelectric records at two stations, Kakioka near Tokyo and Memanbetsu in Hokkaido are examined as typical examples. The whole duration of a disturbance associated with polar substorms is divided into six equal time intervals; the time of its beginning being denoted by $t_{0}$ and that of its end by $t_{6}$, while the midpoint of the duration by $t_{3}$. The variation part of each component is defined approximately as the deviation from the straight line connecting the points at $t_{0}$ and $t_{6}$ on both the magnetograms and geoelectric records. Generally the variations of horizontal components of both the fields are expressed by loops starting from and ending at the origins. In the following a variation diagram is represented by a vector

*Present address: 5-4-58, Fujimi-cho, Higashi-Murayama, Tokyo 189, Japan. 
having the maximum deviation from the origin for the sake of simplicity. In order to find directional characteristics of the fields, geomagnetic variations are classified into eight groups by their variation azimuths, and geomagnetic variation diagram $\Delta R$, the corresponding geoelectric ones $\Delta E$ and the time variation curves of geomagnetic vertical components $\Delta Z$ at the two stations are schematically represented in Fig. 1 where geomagnetic and geoelectric diagrams are shown by solid and broken arrows, respectively, and the amplitudes of $\Delta Z$ are normalized by those of $\Delta R$. It is very remarkable that geoelectric field is not always perpendicular to geomagnetic field, contrary to Maxwell's law. In fact, this was first pointed out by BIRKELAND (1913). In other words, geoelectric field changes in a strongly preferred azimuth peculiar to each station, while geomagnetic field changes in almost all azimuths though there are statistically predominant azimuths depending on types of disturbances and on geomagnetic latitudes of the observation points. This means that the earth crust has strongly anisotropic electric conductivity. In Fig. 1 preferred azimuths in geoelectric changes are denoted by broken arrows that are termed "principal direction" by YOSHIMATSU (1957): the preferred azimuths at Kakioka are roughly east-west

a)

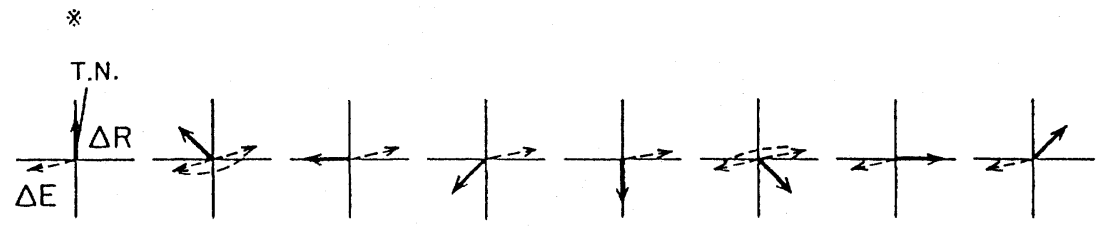

b)
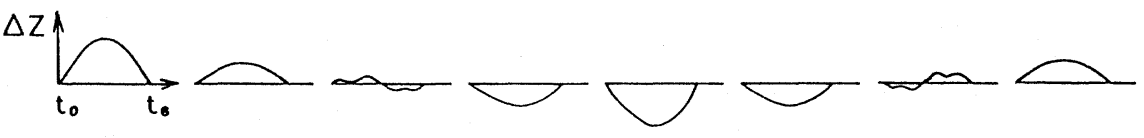

Kakioka

a)
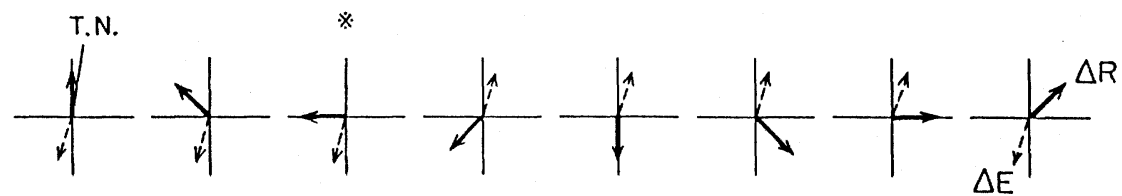

b)
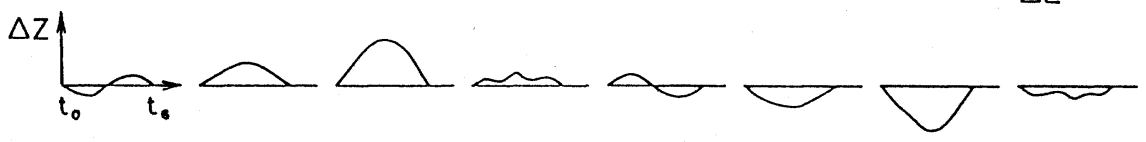

\section{Memanbetsu}

Fig. 1. Schematic representation of geomagnetic and geoelectric variations at Kakioka and Memanbetsu. (a) Variation vectors: Solid arrows $(\Delta R)$ : geomagnetic variation vectors, Broken arrows $(\Delta E)$ : geoelectric variation vectors, T.N.: true north. (b) Temporal changes in geomagnetic vertical components $\Delta Z$. Their amplitudes are normalized by those of $\Delta R$. Asterisks denote the induction arrows and the corresponding preference arrows. At the 2 nd and 6 th variation diagrams of Kakioka, $\boldsymbol{E}$ vectors are transient and may take either directions shown in the figure. 
direction and those at Memanbetsu are roughly north-south direction. Geoelectric changes take either of the principal directions, controlled by the azimuths of geomagnetic changes. However, at some stations, geoelectric changes are unstable or transient between the principal directions of the opposite azimuths for certain azimuths of geomagnetic variations as illustrated by two variation diagrams ( 2 nd and 6th ones) of Kakioka in Fig. 1. Geomagnetic vertical components $\Delta Z$ take the maximum amplitudes when geomagnetic horizontal vectors $\Delta R$ point perpendicularly towards the principal direction at each station: roughly north-south direction at Kakioka and roughly east-west direction at Memanbetsu. The azimuths in which $\Delta Z$ takes the maximum positive values are termed "induction arrows" which are marked by the asterisks in Fig. 1. The lengths of induction arrows represent the ratios of $\Delta Z$ and $\Delta R$, and their directions are the same as "Wiese vectors". The principal directions corresponding to the induction arrows are vectors and termed "preference arrows" in this paper. At the two stations, two vectors, preference arrows and induction arrows are approximately perpendicular to each other.

\section{Poynting Vectors in Geomagnetic and Geoelectric Fields}

In electromagnetic fields energy fluxes are defined by Poynting vectors $\boldsymbol{S}=(c / 4 \pi) \boldsymbol{E} \times \boldsymbol{H} \mathrm{erg} / \mathrm{cm}^{2} \mathrm{sec}$ in Gaussian unit that is adopted here to compare the energy fluxes with the traditional solar constant later. As known well, magnitude of Poynting vector is equal to the product of the vector magnitudes ( $E$ and $H$ ) and the sine of the angle between them, and its direction is perpendicular to the plane of the two vectors and in such a sense that the three vectors form a right-handed set. In this paper, vector products of preference arrows and induction arrows are termed "Poynting arrows" that define the energy flow qualitatively, positive or negative, not quantitatively: Quantitative relationships between geomagnetic and geoelectric changes have been discussed by many researchers. As shown in Fig. 1, during all variations at both the stations, the Poynting arrows are either directed inwards the earth or nearly zero in magnitude, and the geomagnetic vertical components $\Delta Z$ take the maximum amplitudes, positive or negative, when the arrows take the maximum values. The examples of induction arrows and preference arrows observed at some stations in and around Japan (mainly after YOSHIMATSU, 1957; VANYAN et al., 1968; MORI, 1974; YAMASHITA and YOKOYAMA, 1976) are shown in Fig. 2 where the preference arrows point the preferred direction corresponding to the induction arrows. At MZ (Mizusawa) in the northeastern part of Honshu, the induction arrow is nearly zero because its geomagnetic vertical components are always nearly zero in variations of a few hour periods, and consequently the Poynting arrow should be nearly zero regardless of the preference arrow. At some stations in the figure only principal directions of geoelectric field variations were determined and shown by broken lines, not arrows. YOSHIMATSU (1957) summarized the general features of principal directions with respect to the topographies in the vicinity of the stations: The directions near the sea are usually perpendicular to the nearest coastlines while the directions inland are characterized by the general trend of surrounding topographies. 


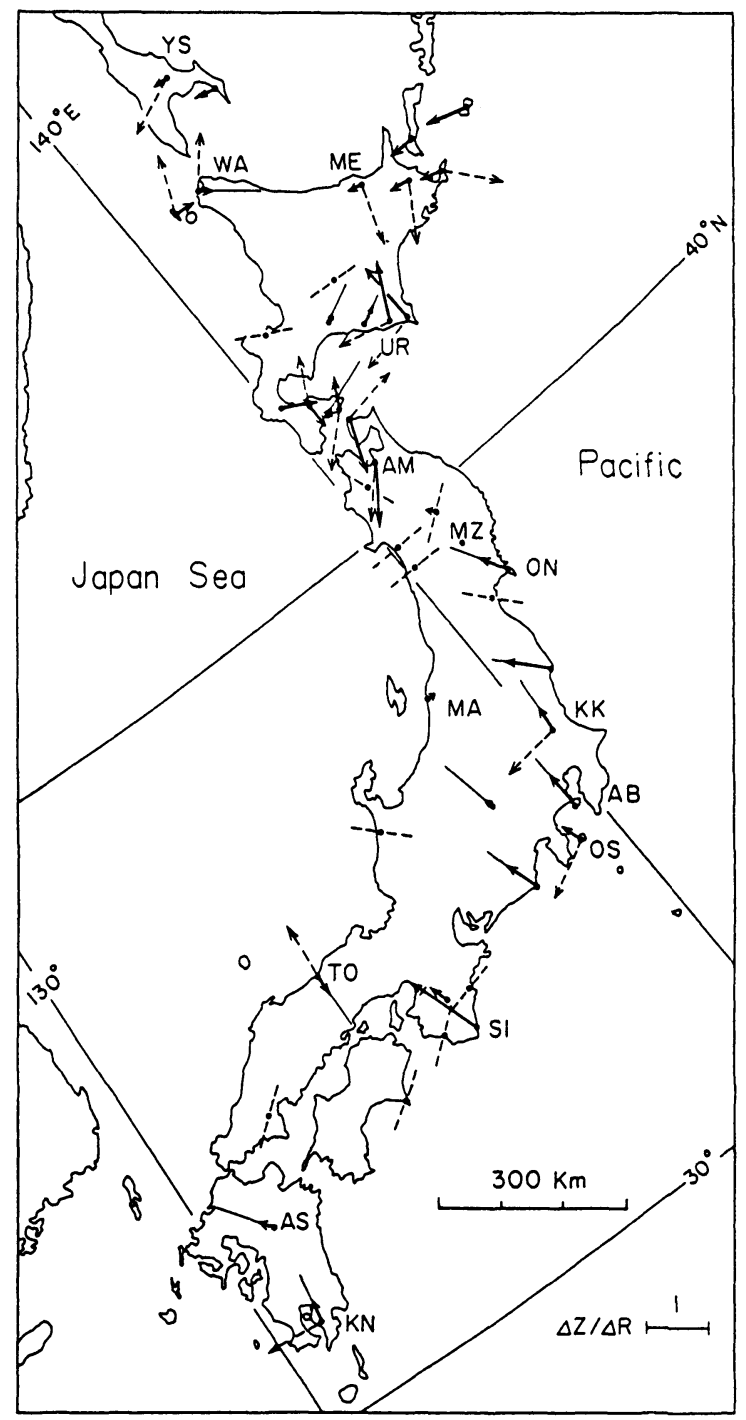

Fig. 2. Distribution of induction arrows (solid) and preference arrows (broken) in and around Japan. Lengths of induction arrows represent the ratios of geomagnetic vertical and horizontal components. Broken lines represent only the preferred directions (principal directions of YOSHIMATSU, 1957) of geoelectric variations. 
Although, in Fig. 2, there are several stations where only one of the pair has been observed, one notices that the Poynting arrows point inwards the earth at WA (Wakkanai), UR (Urakawa), OS (Ooshima) and KN (Kanoya) besides KK (Kakioka) and ME (Memanbetsu), and that those are nearly zero at AM (Asamushi) and TO (Tottori). Due to the effects of the converging or diverging electric currents conducted from other places where the currents were originally induced ("induction-conduction processes"), the inward Poynting arrows are intensified at some stations and weakened at the other stations. Theoretically we may expect that conductivity anomalies could even change the vectors from inward to outward, but we do not find such examples in Fig. 2. Among the stations that show the inward vectors, some stations are effected by conductivity anomalies such as the peninsular effects at UR, the island effects at OS and the upper mantle anomaly effects in addition to the coastline ones at $\mathrm{KK}$ and $\mathrm{AB}$ though we can not distinguish the conductivity anomalies by means of the Poynting arrows. Of the stations that show nearly zero vectors, the AM anomaly was found and interpreted by HASEGAWA (1961) as the effect of anomalously large amplitudes in geoelectric changes there, while the TO anomaly by MIYAKOSHI $(1964,1969)$ as the effect of the sea of Japan located at the north of the station.

For reference, the inward energy flow by a geomagnetic bay-type variation, say $10 \mathrm{nT}$ and $10 \mathrm{mV} / \mathrm{km}$ in amplitude, at a normal station is estimated at $10^{-10}$ $\mathrm{cal} / \mathrm{cm}^{2} \mathrm{~min}$ in order of magnitude, which is far smaller than the solar constant (about $\left.1.9 \mathrm{cal} / \mathrm{cm}^{2} \mathrm{~min}\right)$.

\section{Concluding Remarks}

Vertical components of geomagnetic variations take the maximum amplitudes, whether positive or negative, when Poynting arrows take the maximum values. In other words, when geomagnetic horizontal vectors are directed in such azimuths that electromagnetic inductions work most effectively. Conductivity anomalies either intensify or weaken Poynting arrows according to the peculiarities at respective stations. Hitherto, conductivity anomalies have been studied mainly by means of anomalies of geomagnetic variations. Poynting arrows explicitly reveal that conductivity anomalies are directly correlated with strong anisotropy of electric conductivity in the earth. The qualitative and quantitative studies on "preference arrows" should be desirable.

\section{REFERENCES}

Birkeland, K., The Norwegian Aurola Polaris Expedition, 1902-03, Vol. 1, On the cause of magnetic storms and the origin of terrestrial magnetism, 1st and 2nd Sect., 801 pp., Christiania, H. Aschehoug \& Co., 1913.

Hasegawa, J., Relationships between Anomalous Variations in Earth-Current and Underground Structures, and Their Application to Geophysical Prospectings, Sumitomo Metal Mining Co., 100 pp., 1961 (in Japanese). 
Miyakoshi, J., Geomagnetic and telluric current observation in the San'in district (I), Lib. Arts J., Tottori Univ., 15, 133-138, 1964 (in Japanese).

Miyakoshi, J., Geomagnetic and telluric current observation in the San'in district (II), J. Fac. Gen. Ed., Tottori Univ., 3, 199-204, 1969 (in Japanese).

MORI, T., Observations of geomagnetic and geoelectric variations in the eastern part of Hokkaido, Mem. Kakioka Mag. Obs., 16, 45-58, 1974.

Vanyan, L. L., B. E. Marderfeld, and I. K. Tuyezov, Anomaly in geomagnetic variations in the Far East, Geomag. and Aerono., 8, 574-578, 1968.

Yamashita, H. and I. Yokoyama, Interpretation of the "Northeastern Japan Anomaly" in electrical conductivity of the upper mantle, J. Geomag. Geoelectr., 28, 329-332, 1976.

Yoshimatsu, T., Universal earth-currents and their local characteristics, Mem. Kakioka Mag. Obs., Supp. 1, 1-76, 1957. 